\title{
A micromachined silicon parallel acoustic delay line (PADL) array for real-time photoacoustic tomography (PAT)
}

Young Cho, Cheng-Chung Chang, Lihong V. Wang, Jun Zou

Young Cho, Cheng-Chung Chang, Lihong V. Wang, Jun Zou, "A micromachined silicon parallel acoustic delay line (PADL) array for real-time photoacoustic tomography (PAT)," Proc. SPIE 9323, Photons Plus Ultrasound: Imaging and Sensing 2015, $93232 Z$ (11 March 2015); doi: $10.1117 / 12.2080178$

SPIE. Event: SPIE BiOS, 2015, San Francisco, California, United States 


\title{
A Micromachined Silicon Parallel Acoustic Delay Line (PADL) Array for Real-Time Photoacoustic Tomography (PAT) \\ Young Cho*a , Cheng-Chung Chang ${ }^{\mathrm{a}}$, Lihong V. Wang ${ }^{\mathrm{b}}$, and Jun Zou ${ }^{\mathrm{a}}$ \\ ${ }^{a}$ Texas A\&M University, 3128 TAMU, College Station, TX, USA 77843; ${ }^{b}$ Washington University \\ in St. Louis, 1 Brookings Dr., St. Louis, MO USA 63130
}

\begin{abstract}
To achieve real-time photoacoustic tomography (PAT), massive transducer arrays and data acquisition (DAQ) electronics are needed to receive the PA signals simultaneously, which results in complex and high-cost ultrasound receiver systems. To address this issue, we have developed a new PA data acquisition approach using acoustic time delay. Optical fibers were used as parallel acoustic delay lines (PADLs) to create different time delays in multiple channels of PA signals. This makes the PA signals reach a single-element transducer at different times. As a result, they can be properly received by single-channel DAQ electronics. However, due to their small diameter and fragility, using optical fiber as acoustic delay lines poses a number of challenges in the design, construction and packaging of the PADLs, thereby limiting their performances and use in real imaging applications.

In this paper, we report the development of new silicon PADLs, which are directly made from silicon wafers using advanced micromachining technologies. The silicon PADLs have very low acoustic attenuation and distortion. A linear array of 16 silicon PADLs were assembled into a handheld package with one common input port and one common output port. To demonstrate its real-time PAT capability, the silicon PADL array (with its output port interfaced with a single-element transducer) was used to receive 16 channels of PA signals simultaneously from a tissue-mimicking optical phantom sample. The reconstructed PA image matches well with the imaging target. Therefore, the silicon PADL array can provide a $16 \times$ reduction in the ultrasound DAQ channels for real-time PAT.
\end{abstract}

Keywords: real time, handheld array, photoacoustic tomography, single-crystalline silicon, microfabrication, laser micro-machining, parallel acoustic delay line.

\section{INTRODUCTION}

Photoacoustic tomography (PAT) is a biomedical imaging technique that provides rich optical absorption contrast and deep ultrasonic penetration depth [1-3]. To conduct PAT, a single-element ultrasonic transducer can receive photoacoustic (PA) signals by scanning multiple locations one by one. Although this kind of imaging setup is simple, the data acquisition (DAQ) speed is largely limited by both the pulse repetition rate of the laser and the time lapse necessary for the transducer to scan over all the locations. 1D or 2D ultrasonic transducer arrays, while more complex to construct, allow much faster imaging because they can detect multiple PA signals simultaneously, without mechanical scanning. However, adding ultrasonic transducer elements and DAQ channels increases the system's cost. To address this issue, we originally developed a handheld PAT probe which consisted of two arrays of eight parallel acoustic delay lines (PADLs) made of tightly wound fused-silica optical fiber coils [4]. Each optical-fiber PADL array transmitted eight channels of PA signals from the imaging target to a single-element ultrasonic transducer, while imposing a different acoustic time delay on each PA signal. As a result, the eight time-delayed PA signals reached the single-element ultrasonic transducers at different times and therefore could be received unmixed. A PA image was successfully reconstructed based on the 16 channels of PA signals received by the two ultrasonic transducers and two data acquisition (DAQ) channels, which corresponds to a signal-to-channel reduction ratio of 8:1. However, the use of fused-silica optical fibers as the PADLs presented a few drawbacks. First, to make a compact PAT probe, the optical fibers had to be tightly wound into compact coils, which required tedious and intensive manual assembly. Second, the increased number of contacts with the supporting structures and the bending of the optical fibers in the probe housing could cause higher attenuation and distortion of the PA signals. These two factors limited the number of the PADLs that could be practically built and interfaced to a single-element transducer, and therefore limited the signal-to-channel reduction ratio and field of view of the PAT probe.

Photons Plus Ultrasound: Imaging and Sensing 2015, edited by Alexander A. Oraevsky, Lihong V. Wang

Proc. of SPIE Vol. 9323, 93232Z - (c) 2015 SPIE · CCC code: 1605-7422/15/\$18 doi: $10.1117 / 12.2080178$ 
In this paper, we report the development of a new micromachined silicon PADL array and the initial demonstration of its application in real-time PAT. As an almost perfectly elastic crystal material, single-crystalline silicon has excellent mechanical and acoustic properties. It has extremely low acoustic attenuation in the $\mathrm{MHz}$ range [5], superior to commonly-used low-acoustic-loss materials such as aluminum and silica. With the recent development of advanced micromachining processes, complex silicon microstructures can be directly fabricated on a silicon substrate in one step without intensive manual assembly. Therefore, single-crystalline silicon is structurally superior to optical fibers for the construction of PADLs. In this work, a 16-channel silicon PADL array was designed, fabricated, and assembled, and its acoustic transmission properties were characterized. To demonstrate its PA imaging capability, the silicon PADL array was interfaced with one single-element transducer followed by one channel of DAQ electronics to receive 16 channels of PA signals simultaneously. A PA image of an optically-absorbing target embedded in an optically-scattering phantom was reconstructed, which matched well with the actual size of the imaging target. The silicon PADL array allowed a signal-to-channel reduction ratio of 16:1, two times higher than that of the optical fiber PADL array demonstrated previously $[4,6]$. Therefore, this new acoustic time delay approach could significantly simplify the design and construction of ultrasonic receivers for real-time PAT.

\section{DESIGN AND CONSTRUCTION}

\subsection{Design}

Due to the high acoustic velocity $(\sim 8430 \mathrm{~m} / \mathrm{s}$ [7]) of silicon, an acoustic delay length ranging from a few to tens of centimeters is necessary to provide suitable acoustic time delays $(1-10 \mu \mathrm{s})$ between two adjacent PADLs, so that the PA signals do not overlap with each other and therefore can be clearly distinguished when they reach the single-element transducer [8]. To achieve a compact structure, the PADLs were designed as tightly wound spiral coils (Figure 1a). Table 1 lists the main design parameters of the silicon PADL array. The shortest and longest acoustic time delays are 9.5 $\mu \mathrm{s}$ and $107 \mu \mathrm{s}$, respectively. The nominal incremental acoustic time delay between any two adjacent channels is $6.5 \mu \mathrm{s}$, which corresponds to a penetration depth of $\sim 1 \mathrm{~cm}$ in soft tissue (assuming an acoustic velocity of $\sim 1500 \mathrm{~m} / \mathrm{s}$ ). The cross sectional area of the silicon PADLs is $500 \mu \mathrm{m} \times 250 \mu \mathrm{m}$, which provides good structural stability and also good acoustic signal transmission for PA signals. For a rectangular acoustic delay line, its width or thickness $(d)$ (whichever is smaller) should satisfy $\left(d f / V_{0}\right)<<1$ to avoid signal distortion due to higher-order mode propagation and mode dispersion, where $f$ is the frequency of the signal, and $V_{0}$ is the acoustic velocity of the delay-line material [9]. Assuming the silicon PADLs are interfaced with a single-element ultrasonic transducer with a center frequency of $2.25 \mathrm{MHz}$ and a bandwidth of $100 \%$, the maximum detection frequency will be $3.375 \mathrm{MHz}$. This value yields a $d f / V_{0}$ ratio of $0.1(<<1)$ to ensure single-mode transmission of the PA signals with minimal mode dispersion. Based on our previous simulation and experimental characterization results [8], the smallest radius of curvature in the center portion of the PADL structure was made larger than $2 \mathrm{~mm}$ to ensure high acoustic transmission efficiency by reducing mode conversion at curved sections of the acoustic delay lines. Figure 1a shows the schematic design of the longest (the $16^{\text {th }}$ ) PADL. The diameter of its circular portion is $30 \mathrm{~mm}$, which is still compact even though its delay length reaches $90 \mathrm{~cm}$. Figure $1 \mathrm{~b}$ shows a schematic of the 16-channel silicon PADL array.

Table 1. Main design parameters of silicon PADLs

\begin{tabular}{ccccccc}
\hline $\begin{array}{c}\text { Number of } \\
\text { channels }\end{array}$ & $\begin{array}{c}\text { Shortest } \\
\text { delay }\end{array}$ & $\begin{array}{c}\text { Longest } \\
\text { delay }\end{array}$ & $\begin{array}{c}\text { Incremental } \\
\text { delay }\end{array}$ & $\begin{array}{c}\text { Center } \\
\text { frequency }\end{array}$ & Cross section & $\begin{array}{c}\text { Minimum radius of } \\
\text { curvature }\end{array}$ \\
\hline 16 & $9.5 \mu \mathrm{s}$ & $107 \mu \mathrm{s}$ & $6.5 \mu \mathrm{s}$ & $2.25 \mathrm{MHz}$ & $500 \mu \mathrm{m} \times 250 \mu \mathrm{m}$ & $2 \mathrm{~mm}$ \\
\hline
\end{tabular}

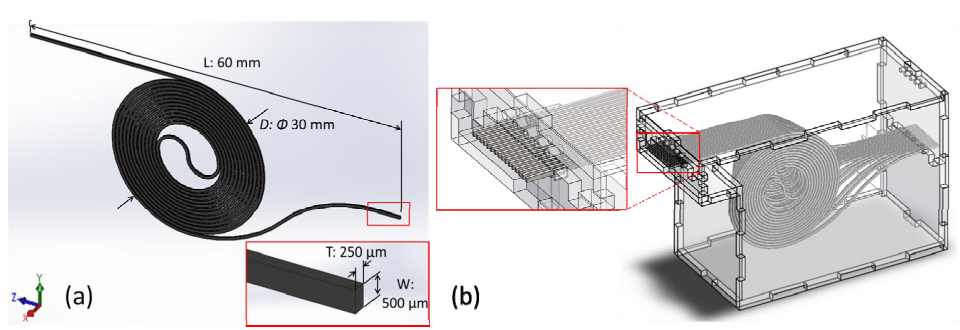

Figure 1. (a) 3D schematic design of the longest $\left(16^{\text {th }}\right)$ silicon PADL. (b) The assembly of 16-channel silicon PADL array and a zoomed-in view of the input port. 


\subsection{Fabrication and Assembly}

The silicon PADLs were fabricated on 4-inch $\{100\}$-oriented single crystal silicon wafers with a thickness of $250 \mu \mathrm{m}$ (University Wafers, Boston, MA, USA) by using a microfabrication process developed previously [8]. To precisely position the silicon PADLs in the array at the input and output ports, two identical spacer structures were laser cut from a $0.4 \mathrm{~mm}$ thick clear acrylic sheet (Universal Laser Systems, PLS6.75) (Figure 2a). Each spacer structure consists of 16 shallow trenches $(300 \mu \mathrm{m} \times 500 \mu \mathrm{m})$ to support the silicon PADLs and 15 interspaced deep trenches $(200 \mu \mathrm{m} \times 1000$ $\mu \mathrm{m})$ to reduce acoustic crosstalk between adjacent supports. Both the shallow and deep trenches were formed by using a dicing saw. To protect the entire silicon PADL array, an acrylic housing was also fabricated (see Figure 1b). Its top panel, bottom panel, and two side walls were laser cut from a $1.5 \mathrm{~mm}$ thick acrylic sheet. Figure $2 \mathrm{~b}$ shows the fully assembled 16-channel silicon PADL array.
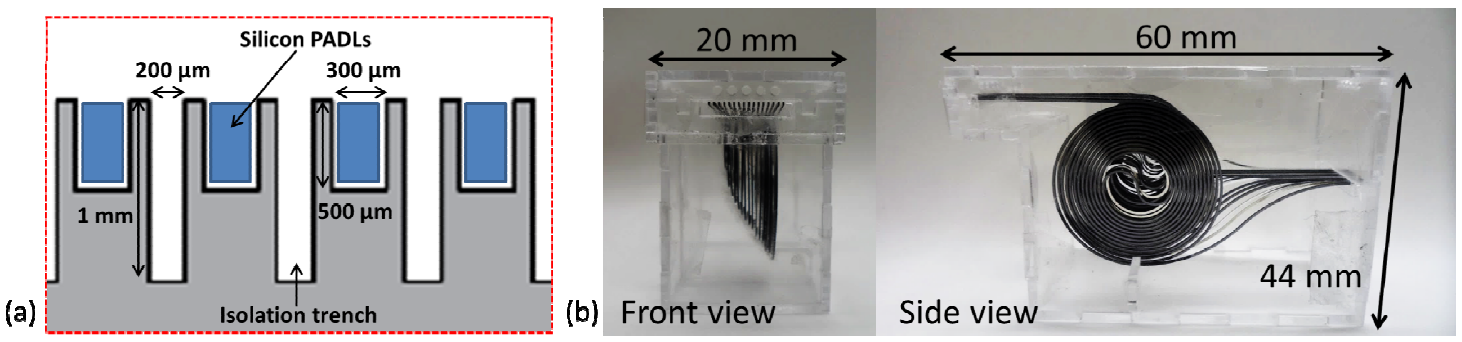

Figure 2. (a) Zoomed-in view of spacers with dimension. (b) Fully assembled 16-channel silicon PADL array.

\section{PA IMAGING}

\subsection{PA imaging setup}

Figure 3a shows the experimental setup of the PA imaging experiment. A frequency-doubled Q-switched 532-nm Nd:YAG laser (LT-P100, Mychway Technology Co., Limited, Shenzhen, Guangdong, China) was the light source. The laser pulse duration was $8 \mathrm{~ns}$ and the pulse repetition rate was $5 \mathrm{~Hz}$. The imaging target was a piece of silicon $(1 \mathrm{~mm} \times$ $0.25 \mathrm{~mm} \times 1 \mathrm{~mm})$ embedded in an optical tissue phantom $(100 \mathrm{~mm} \times 100 \mathrm{~mm} \times 50 \mathrm{~mm})$ (Figure 3b). The phantom was composed of $2 \%$ agar gelatin by weight, and the target was located $2.5 \mathrm{~mm}$ below the phantom surface. The PA signals generated from the imaging target propagated along the silicon PADL array and were received by a single-element 2.25 MHz ultrasonic transducer (V106, Olympus NDT, Waltham, MA, USA), then amplified by a pulser/receiver (5072PR, Olympus NDT, Waltham, MA, USA). The amplified PA signals were averaged five times and then recorded on a digital oscilloscope (TDS2014B, Tektronix Inc., Beaverton, OR, USA) at a sampling rate of $5 \mathrm{MHz}$.

(a)

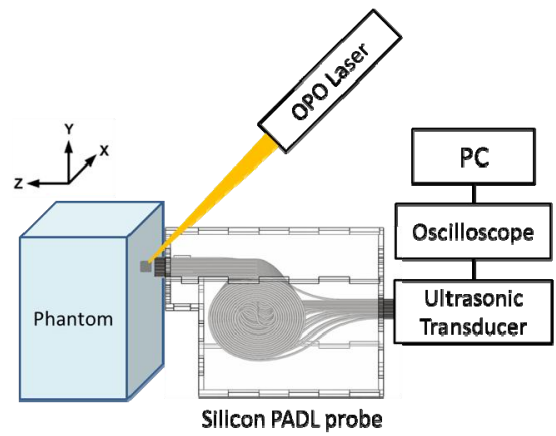

(b)

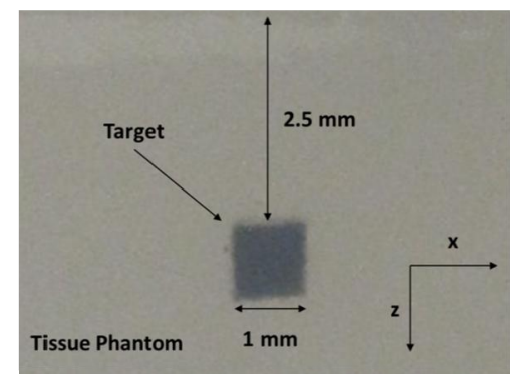

Figure 3. (a) PA imaging setup with the 16-channel silicon PADL array (b) Photograph of an optically absorbing target embedded in optically scattering medium.

\subsection{PA data acquisition and image reconstruction}

The PA signals were averaged five times to generate one PA image; thus the imaging speed was $1 \mathrm{~Hz}$. The end of each PADL on the sample surface was considered as a single element in an ultrasound array. Each sequentially detected PA signal was reshaped into 16 separate PA signals, based on the predefined time positions measured by the ultrasound 
transmission experiment. To reconstruct PA images, a commonly used delay-and-sum beamforming method was applied [10]. The PA images were reconstructed by summing the data from all 16 channels after compensating for the time delays in each silicon PADL, based on its length and matching phase. In a two-dimensional beam field along the $\mathrm{x}$ and $\mathrm{z}$ directions, the time delay $\tau_{n}$ for the $n^{\text {th }}$ PADL located on $\left(x_{n}, z_{n}\right)$ can be calculated as follows:

$$
\tau_{n}=\frac{\sqrt{\left(x-x_{n}\right)^{2}+\left(z-z_{n}\right)^{2}}-R}{c},
$$

where $R$ is the distance from the focal point to the center of the delay line, and $c$ is the speed of sound in soft tissue, 1540 $\mathrm{m} / \mathrm{s}$. Envelope detection was applied by using Hilbert transformation along the axial direction (the $z$ direction), followed by taking the absolute value.

\section{RESULTS AND DISCUSSION}

Figure 4 shows the 16 PA signals from the silicon PADL array acquired by the single-element ultrasound transducer and the DAQ channel after five times averaging. Each PA signal was identified based on the time delay ( $7 \mu \mathrm{s})$ previously determined in ultrasound transmission testing. Figure 5 shows the reconstructed PA image in Matlab ${ }^{\circledR}$. The envelope of the PA signals was detected by using Hilbert transformation, and the PA image was reconstructed using the synthetic aperture focusing technique (SAFT) [11] to improve spatial resolution. As shown in Figure 5a, in the reconstructed PA image, both the location and the size of the imaging target match the actual values. The image contrast, defined as $\left(P A_{\text {target }}-P A_{\text {background }}\right) / P A_{\text {background }}$, is calculated to be $\sim 1.9$. The spatial resolution, defined as the one-way distance between $10 \%$ and $90 \%$ of the maximum, divided by the minimum, is $\sim 2.1 \mathrm{~mm}$. Figure $5 \mathrm{~b}$ shows the enhanced PA image after applying a signal threshold of $30 \%$ (Figure $5 \mathrm{~b}$ ).
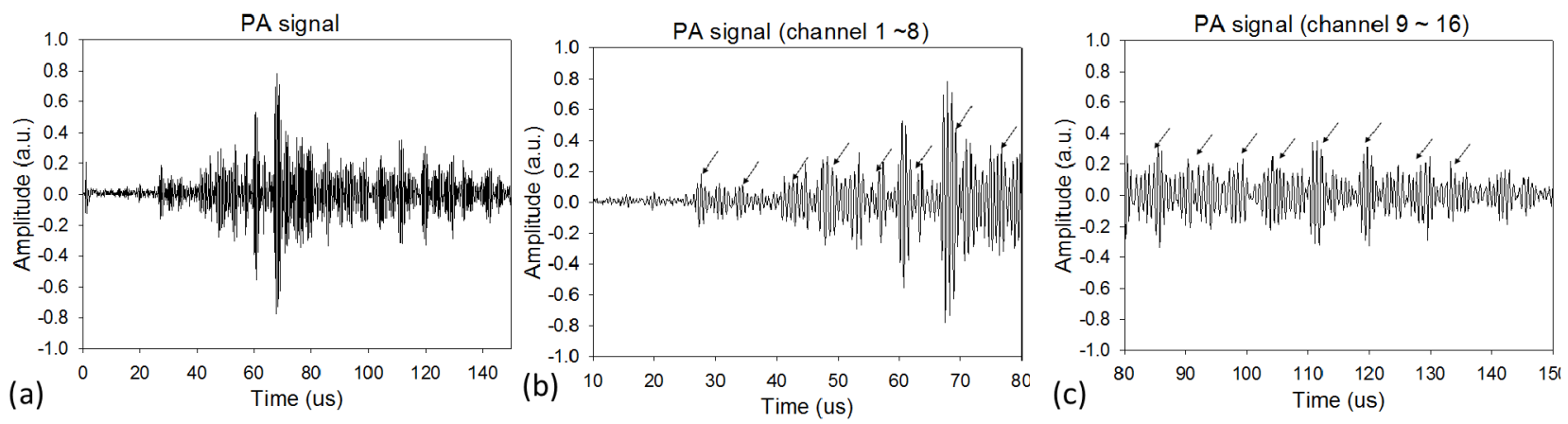

Figure 4. (a) PA signals received by the ultrasonic transducer through the 16 silicon PADLs. (b) Zoomed-in view of the eight PA signals from channels 1 to 8. (c) Zoomed-in view of the eight PA signals from channels 9 to 16.
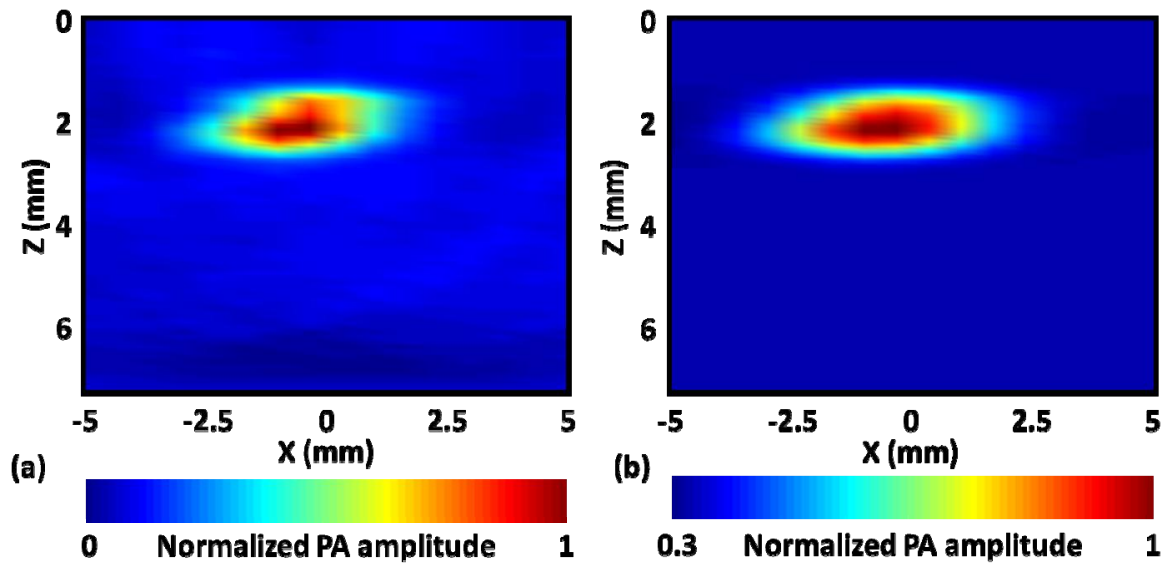

Figure 5. (a) Reconstructed PA image of the imaging target. (b) Thresholded(30\%) PA image of (a). 


\section{CONCLUSION}

In this work, we have successfully demonstrated a micromachined silicon PADL array for real-time PAT. The array capitalizes upon the extremely low acoustic loss of single-crystalline silicon and the high precision of the micromachining process. Using the silicon PADL array, a transducer/channel reduction ratio of 16:1 was achieved, which is twice that obtained with optical-fiber PADLs. In the future, we will investigate larger silicon PADL arrays to provide even higher transducer/channel reduction ratios to fulfill the potential of the time-delayed ultrasound reception approach. In addition, we will also improve the acoustic collection angle and impedance matching at the input terminals to obtain better PA signal detection.

Acknowledgement: This work was supported in part by a grant (CMMI-1131758) from the National Science Foundation to J.Z. and a grant (DP1 EB016986) from the National Institutes of Health to L.V. Wang has a financial interest in Microphotoacoustics, Inc. and in Endra, Inc., which, however, did not support this work.

\section{REFERENCES}

[1] Oraevsky, A., and Karabutov, A., [Biomedical Photonics Handbook], CRC Press, Boca Raton, Florida, 34/1 - 34/34 (2003).

[2] Wang, L., [Photoacoustic imaging and spectroscopy], CRC Press, Boca Raton, Florida (2009)

[3] Kim, C., Favazza, C., and Wang, L., "In vivo photoacoustic tomography of chemicals: high-resolution functional and molecular optical imaging at new depths," Chem. Rev., 110(5), 2756-2782(2010).

[4] Cho, Y., Chang, C., Yu, J., Jeon, M., Kim, C., Wang, L., and Zou, J., "Handheld photoacoustic tomography probe built using optical-fiber parallel acoustic delay lines," J. Biomed. Opt., 19(8), 086007(2014).

[5] Auld, B., [Acoustic fields and waves in solids], Wiley, New York(1973).

[6] Yacipi, M., Kim, C., Chang, C., Jeon, M., Guo, Z., Zou, J., and Wang, L., "Parallel acoustic delay lines for photoacoustic tomography," J. Biomed. Opt., 17(11), 116019(2012).

[7] Cheeke, J., [Fundamentals and applications of ultrasonic waves], CRC Press, New York, 453(2012).

[8] Chang, C, Cho, Y., Wang, L., and Zou, J., "Micromachined silicon acoustic delay lines for ultrasound applications," J. Micromech. Microeng., 23(2), 025006(2013).

[9] Meeker, T., "Dispersive ultrasonic delay lines using the first longitudinal mode in a strip," IRE Trans. Ultrason. Eng., 7, 53-58(1960).

[10] Xu, M. and Wang, L., "Universal back-projection algorithm for photoacoustic computed tomography," Phys. Rev., 71(1), 016706(2005).

[11] Yiltalo, J. and Ermert, H., "Ultrasound synthetic aperture imaging: monostatic approach," IEEE Trans. Ultrason. Ferroelectr. Freq. Control., 41(3), 333-339(1994). 